\title{
Recurrent Alveolar Rhabdomyosarcoma
}

National Cancer Institute

\section{Source}

National Cancer Institute. Recurrent Alveolar Rhabdomyosarcoma. NCI Thesaurus. Code C142881.

The reemergence of alveolar rhabdomyosarcoma after a period of remission. 